\title{
Shedding Light on Dark Matter: A Faraday Rotation Experiment to Limit a Dark Magnetic Moment
}

\author{
Susan Gardner \\ Center for Particle Astrophysics and Theoretical Physics, \\ Fermi National Accelerator Laboratory, Batavia, IL 60510 and \\ Department of Physics and Astronomy, \\ University of Kentucky, Lexington, KY 40506-0055*
}

\begin{abstract}
A Faraday rotation experiment can set limits on the magnetic moment of a electrically-neutral, dark-matter particle, and the limits increase in stringency as the candidate-particle mass decreases. Consequently, if we assume the dark-matter particle to be a thermal relic, our most stringent constraints emerge at the keV mass scale. We discuss how such an experiment could be realized and determine the limits on the magnetic moment as a function of mass which follow given demonstrated experimental capacities.
\end{abstract}

\footnotetext{
${ }^{*}$ Permanent Address
} 


\section{INTRODUCTION}

Disparate astronomical observations provide compelling evidence for additional, nonluminous matter, or dark matter, in gravitational interactions. The evidence includes the persistence of the galactic rotation curves to distances for which little luminous matter is present [1, 2], the relative strength and shape of the galaxy-distribution power spectrum at large wave numbers [3], and the pattern of acoustic oscillations in the power spectrum of the cosmic microwave background [4]. The cosmological evidence, in aggregrate, points to the assessment that dark matter comprises some twenty-three percent of the energy density of the universe, with a precision of a few percent [5]. Yet we know little about the nature of dark matter - we do not know its mass, its quantum numbers, or even with surety that it is indeed composed of isolated elementary particles. A recent gravitational lensing study does disfavor a modification of gravity in explanation of its effects [6]: we shall assume [7] that dark matter exists and is matter.

We do know other things about dark matter [8, 9]; namely, that it is not hot [10], and that it appears to lack both electric and color charge [11, 12, 13, 14, 15, 16]. Here we use temperature, i.e., whether dark matter is "cold" or "hot," to connote whether dark matter is non-relativistic or relativistic, respectively, at the redshift at which it decouples from matter in the cooling early Universe. For so-called thermal relics, this criterion selects the mass of the dark candidate as well, so that colder particles are heavier. However, alternative production scenarios can exist, and very light particles can also act as cold dark matter, as in the case of the axion [18]. Nevertheless, model-dependent constraints do exist on the mass of the candidate particle, and we consider them in Sec. II.

The evidence for dark matter emerges from astrophysical observations of gravitational interactions, but establishing its couplings to Standard Model (SM) particles has proven elusive. Nevertheless, such a quest is of great import, for it is through such means that its mass and quantum numbers can ultimately be determined. Indeed all direct and indirect means of detecting dark matter rely on the notion that it does indeed experience weak or electromagnetic interactions to some degree. Turning to the known particles of the SM for guidance, the neutron shows us that a particle can have both a vanishing electric charge and a significant magnetic moment. Thus we wish to constrain the possibility that dark matter has a small electromagnetic coupling, via its magnetic moment. We review the existing constraints on this possibility in Sec. III.

In this paper we consider a new technique for the direct detection of dark matter, namely, through use of the gyromagnetic Faraday effect [19]. Alternatively, this effect can be used to limit a possible magnetic moment $\mu$ of a dark-matter particle of mass $M$. Let us consider how this could work. An electrically neutral medium of particles which possesses a net magnetization in an external magnetic field is circularly birefringent, even if the medium is isotropic. This implies that the propagation speed of light in the medium depends on the state of its circular polarization, so that light prepared in a state of linear polarization will suffer a rotation of the plane of that polarization upon transmission through the medium [20, 21], as long as it does not travel at right angles to the external magnetic field. We term this the gyromagnetic Faraday effect, after Ref. [22]. Note that we need not rely on any existing magnetization of the dark matter in order to realize an effect. Rather we imagine a Faraday rotation experiment mounted in a region with a large external magnetic induction $\boldsymbol{B}_{0}$ and dark matter of spin $S$ incident on it in the direction of $\boldsymbol{B}_{0}$. If the value of $\mu B_{0}$ is larger than the dark matter particle's kinetic energy in the Earth's rest frame, then the field region 
acts as a spin-filter, or longitudinal Stern-Gerlach, device — at least the highest energy spin configuration cannot enter the field region. This technique is used to polarize ultra-cold neutrons (UCNs) in the UCNA experiment at Los Alamos with near 100\% efficiency [23]. Thus, viewed in the Earth's rest frame, the dark matter which sweeps through the field region can possess a net magnetization. If light transits this medium in the direction of the magnetic field, and if we define $k_{ \pm}$to be the wave number for states with right- $(+)$or left-handed $(-)$ circular polarization, then the rotation linearly polarized light suffers in its transit through the medium is given by the angle $\phi=\left(k_{+}-k_{-}\right) l / 2$, where $l$ is the length of transmission through the medium. If $\phi$ is non-zero once all systematic effects which could mimic the signal are excluded, we have evidence for dark matter with a non-zero magnetic moment.

The direct detection of dark matter with a magnetic moment could be realized in a variety of ways. For example, one could search for anomalous recoil events in scattering from nuclei, in just the manner one searches for spin-independent and spin-dependent dark matter-nucleon interactions [24, 25, 26]. Indeed, an experimental signal of the latter, from the DAMA experiment [27, has been interpreted as a limit on a putative dark-matter magnetic moment, namely of $\mu<1.4 \cdot 10^{-4} \mu_{N}$ for a dark matter candidate of $100 \mathrm{GeV}$ in mass [28]. In such experiments, however, the light dark-matter candidates we discuss give rise to momentum transfers which are much too small to be detected, even if the channeling effect proposed by Ref. [29] and studied in Ref. [30] is operative for the events studied in the DAMA/LIBRA NaI detector [31] - note Ref. [32] for further discussion. Alternatively, a dark matter magnetic moment can be found through either nuclear magnetic resonance (NMR) or Faraday rotation studies. A NMR signal is typically realized through the detected change in magnetic field incurred through a spin-flip transition of the magnetic-momentcarrying particle, induced by an applied radio frequency. However, very small magnetic fields are more efficiently discovered through magnetoptical studies [33, 34]. Thus we focus on the use of Faraday rotation to detect dark matter. Precision optical rotation studies have also been conducted in which the external magnetic field is oriented at right angles to the direction of the propagating light, to the end, e.g., of testing the optical birefringence of the vacuum, or, alternatively, of limiting the photon-axion coupling [35, 36]. Such an experimental configuration does not support a nonzero Faraday effect, yet the empirical parameters used in these studies are useful to us, for we employ them to estimate the limit on the magnetic moment as a function of mass in the set-up we consider.

A great variety of dark matter candidates, consistent with the various astrophysical constraints, exist in the literature [37, 38. Indeed, their masses vary from some $10^{-32}$ to $10^{15}$ $\mathrm{GeV}$, and their interaction cross sections — with nucleons — vary over orders of magnitude as well. Typical direct detection strategies rely on the observation of "anomalous" nuclear recoils [25, 26, 38], so that their sensitivity is typically to candidates of $\mathcal{O}(100 \mathrm{GeV})$ in mass scale. The experiment we suggest is sensitive to a completely different window in parameter space - to dark-matter candidates of crudely $\mathcal{O}(1 \mathrm{MeV})$ or less in mass. Although constraints can be set through cosmological studies [19], terrestrial studies are amenable to better control, for the existence of cosmological magnetic fields have not yet been established [39].

Let us conclude our introduction by outlining the sections to follow. We begin by describing, in Secs. II and III, respectively, the mass and magnetic moment constraints which exist on a light dark-matter candidate with a non-zero magnetic moment. We then proceed to review the gyromagnetic Faraday effect in Sec. IV] and to describe in concrete terms the 
experimental limits on $\mu$ one might possibly attain in Sec. V. We conclude with a summary in Sec. VI.

\section{MASS CONTRAINTS}

In standard Big-Bang cosmology, the nature of dark matter impacts the formation of the large-scale structure of the Universe. In particular, if dark matter is cold and collisionless, then galaxy formation proceeds via a hierarchial clustering, namely, from the merging of small protogalactic clumps, on ever larger scales [40, 41, 42, 43]. In contrast, if dark matter is hot, the hierarchy is inverted, so that large protogalactic disks form first, which then clump [41, 42. Galaxies, however, are observed at much larger redshifts than such simulations predict [41, 42. Moreover, observations of particular classes of quasar absorption lines, the so-called damped Lyman- $\alpha$ systems, thought to be the evolutionary progenitors of galaxies today, also favor the former scenario [44. It has also been argued that hot dark matter, i.e., most notably, light, massive neutrinos, cannot explain the galactic rotation curves [45]. However, the cold-dark-matter paradigm does have difficulties in confronting small-scale structure; it yields, in effect, too much clumpiness below the Mpc scale. Warm dark matter has been advocated as a way to alleviate these difficulties [46]. Limits on the mass of warm dark matter emerge from the comparison of the observations of the Lyman- $\alpha$ absorption spectrum with numerical simulations [47, 48, 49, 50]; the limits depend on the particle considered, and the manner in which it is produced [51], yielding [50], at $2 \sigma, M \gtrsim 4$ $\mathrm{keV}$ for a thermal relic and $M \gtrsim 28 \mathrm{keV}$ for a massive sterile neutrino 52 .

Cosmological constraints also exist on the mass of a dark-matter particle. If the particles annihilate via the weak interaction, then $\sigma_{a n n} v$ is parametrically set by $\mathcal{N}_{A} G_{F}^{2} M^{2}$, where $G_{F}$ is the Fermi constant, $\mathcal{N}_{A}$ is a dimensionless factor, and we assume $\sigma_{a n n} \propto 1 / v$. In this case avoiding a dark-matter abundance in excess of the observed relic density bounds $M$ from below. Indeed, under these conditions the mass of the cold dark-matter particle must exceed $\mathcal{O}(2 \mathrm{GeV})$ to avoid closing the Universe [53]. The resulting lower bound on $M$ can be relaxed in different ways. Feng and Kumar [54], e.g., have emphasized that the appearance of $G_{F}$ in $\sigma_{a n n} v$ is simply parametric, that $G_{F}$ can be replaced with $g_{\text {eff }}$, and that the effective coupling $g_{\text {eff }}$ can be small without having the precise numerical value of $G_{F}$. Thus if $g_{\mathrm{eff}}>G_{F}$, the bound on $M$ is weakened. Indeed, such considerations permit dark matter candidates which confront the relic density and big-bang nucleosynthesis constraints successfully but yet range from the $\mathrm{keV}$ to the $\mathrm{TeV}$ scale in mass [54, 55].

In this paper we consider what empirical constraints can be placed on the magnetic moment of a dark matter particle. This hypothesis gives rise to a new annihilation mechanism, though both a dark matter particle and its antiparticle must be present to realize it. We recall that particles with magnetic moments are invariably described by complex field representations, so that such a particle and its anti-particle are physically distinct — by the CPT theorem we expect the magnetic moments of such particles to differ only in sign. If the particle-antiparticle annihilation is mediated by a magnetic moment interaction, then $\sigma_{a n n} v$ is parametrically set by $\mathcal{N}_{A}^{\prime} \alpha^{2} \mu^{2}$ with $\mathcal{N}_{A}^{\prime}$ a dimensionless parameter, as long as $M \gtrsim m_{e}$, the electron mass. The annihilation of still lighter mass dark matter candidates follows a different parametric form. That is, if the particles annihilate to Standard Model particles, then $\sigma_{a n n} v$ is suppressed by higher powers of the coupling constant - at least. Alternatively, if they annihilate to "secluded" dark matter particles, note, e.g., Ref. [56], then $\sigma_{a n n} v$ is of form $\mathcal{N}_{A}^{\prime} \alpha \alpha^{\prime} \mu^{2}$, where $\alpha^{\prime}$ is the electromagnetic coupling of the secluded particles and $\mathcal{N}_{A}^{\prime}$ 
is a dimensionless parameter. Generally, we expect the magnetic moment $\mu$ to be of form $\mu=\kappa e \hbar / 2 M$, where $\kappa$ is the anomalous magnetic moment, so that $\sigma_{\text {ann }} v$ scales as $1 / M^{2}$. The presence of an additional annihilation mechanism should make the light dark matter candidates we consider, of $\mathcal{O}(\mathrm{keV})$ scale in mass, say, decouple as matter and not radiation, so that constraints on the number of relativistic degrees of freedom during the epoch of bigbang nucleosynthesis also do not apply. This new annihilation mechanism becomes more effective as the candidate mass grows lighter. Nevertheless it is still possible to saturate the dark-matter density with such a candidate particle, for the efficacy of the annihilation process can be mitigated by a particle-antiparticle excess [57, 58]. Thus the dark matter relic density need not bound the magnetic moment from above. Moreover, we emphasize that dark matter could have multiple components, so that an upper bound on the magnetic moment could also be evaded by diluting the magnetic-moment-carrying particle with other sorts of dark matter. We shall assume these various annihilation mechanisms are effective enough to permit dark matter candidates as light as $\mathcal{O}(1 \mathrm{keV})$ in mass.

The intensity and morphology of galactic positron emission, as studied by the INTEGRAL satellite [59], has prompted much discussion of dark matter candidates with electromagnetic interactions [54, 60, 61, 62, 63, 64, 65, 66], which are of $\mathcal{O}(1 \mathrm{MeV})$ scale in mass, as well as of other possibilities, as, e.g., in Refs. [67, 68, 69, 70]. If the pattern of the INTEGRAL spectra are indeed explained by dark matter, then additional constraints follow on its nature. For example [61, 62], observational constraints on the diffuse photon flux also impose limits on the mass of the dark-matter candidate $\chi$ through internal bremsstrahlung corrections to the annihilation process $\chi \chi \rightarrow e^{+} e^{-}$. Such constraints apply to our scenario as well; the upshot is that the dark particle's mass is limited to be less than a few MeV [61, 62]. In our case internal bremsstrahlung contributions can also be generated by the magnetic-momentcarrying particle. However, we note that soft photon emission via a M1 transition of an isolated magnetic dipole is slow compared to the rate set by the inverse age of the universe, as we discuss in greater detail in Sec. IV] so that no meaningful limit follows on its magnetic moment. In what follows we consider candidate particles which range from $\mathcal{O}(1 \mathrm{keV})$ to $\mathcal{O}(100 \mathrm{MeV})$ in mass, though our limits are most effective at sub-MeV mass scales.

\section{MAGNETIC MOMENT CONTRAINTS}

Various constraints on the magnetic moment of a dark-matter particle for masses in excess of $1 \mathrm{MeV}$ have been considered in Ref. [58]. We review these and more, in order to provide a context for the direct detection experiment we suggest. In the mass window of interest to us, two experimental constraints are important - one comes from precision electroweak measurements [58], and the other comes from low-energy $e^{+} e^{-}$collider data, namely from the process $e^{+} e^{-} \rightarrow \nu \bar{\nu} \gamma[71,72]$. This last constrains the magnetic moment of the invisible particle directly and thus offers a constraint on a dark-matter magnetic moment as well. The authors of these studies use data at center-of-mass energies sufficient to produce a $\nu_{\tau} \bar{\nu}_{\tau}$ pair, given accelerator constraints on its mass [71] - this easily includes our mass range of interest. Interpreting their results as a limit on the anomalous magnetic moment of the tau neutrino, the low-energy analyses conclude $\mu_{\nu_{\tau}}<4 \cdot 10^{-6} \mu_{B}$ at 90\% CL [71], and $\mu_{\nu_{\tau}}<9.1 \cdot 10^{-6} \mu_{B}$ at $90 \%$ CL [72] from distinct data sets. A more severe limit on the $\nu_{\tau}$ magnetic moment does exist [73] however, the nature of the $e^{+} e^{-}$limits allows us to interpret them in a manner useful to our current study. For a discussion of how low-energy $e^{+} e^{-}$collider data can probe particular MeV dark matter models [60], see Ref. [74]. 
Precision electroweak measurements also constrain the magnetic moment [58]. The quantity $\Delta \hat{r}$ captures radiative corrections to the relationship between the fine-structure constant $\alpha$, the Fermi constant $G_{F}$, and the $W^{ \pm}$and $Z$ masses, $M_{W}$ and $M_{Z}$ [75]. The difference between the empirically determined value of $\Delta \hat{r}$ and that computed in the Standard Model provides a window $\Delta \hat{r}^{\text {new }}$ to which a dark-matter particle can contribute. Following Refs. [58, 76], we assume $\Delta \hat{r}^{\text {new }}$ is given by the vacuum polarization correction to the photon self-energy from a dark-matter particle with a magnetic moment, with no other adjustments. We choose to study the quantity $\Delta \hat{r}$ as its uncertainty is dominated by that in the running of $\alpha$ [77]. Thus we consider [75]

$$
M_{W}^{2}=\frac{\pi \alpha}{\sqrt{2} G_{F}} \frac{1}{\hat{s}_{z}^{2}(1-\Delta \hat{r})}
$$

where $\hat{s}_{z}$ is computed in the $\overline{\mathrm{MS}}$ scheme and is $\left(1-M_{W}^{2} / M_{Z}^{2}\right)$ up to small corrections. To compute $\Delta \hat{r}^{\text {new }}$, we first recall the general form of the electromagnetic vertex with Dirac and Pauli form factors [78, namely, $\Gamma^{\mu}(k+q, k)=\gamma^{\mu} F_{1}\left(q^{2}\right)+i \sigma^{\mu \nu} q_{\nu} F_{2}\left(q^{2}\right) / 2 M$, where in our case $F_{1}=0$ and $F_{2}=\kappa$. Using the conventions of Ref. [78], we introduce the polarization tensor

$$
i \Pi_{2, t t}^{\mu \nu}(q)=\kappa^{2} e^{2} \int \frac{d^{4} k}{(2 \pi)^{4}} \operatorname{tr}\left\{\sigma^{\mu \alpha} \frac{q_{\alpha}}{2 M} \frac{(\not k+m)}{\left(k^{2}-M^{2}\right)} \sigma^{\nu \beta} \frac{q_{\beta}}{2 M} \frac{(\not k+\not k+m)}{\left((k+q)^{2}-M^{2}\right)},\right\},
$$

with $\sigma^{\mu \nu} \equiv i\left[\gamma^{\mu}, \gamma^{\nu}\right] / 2$. Noting $\Pi_{2, t t}^{\mu \nu}(q)=\left(q^{2} g^{\mu \nu}-q^{\mu} q^{\nu}\right) \Pi_{2, t t}\left(q^{2}\right)$ and

$$
\Delta \hat{r}^{\text {new }}=\Pi_{2, t t}\left(M_{Z}^{2}\right)-\Pi_{2, t t}(0)-M_{Z}^{2}\left(\left.\frac{\partial \Pi_{2, t t}\left(k^{2}\right)}{\partial k^{2}}\right|_{k^{2}=0}\right),
$$

we use standard techniques [78] to determine

$$
\Delta \hat{r}^{\text {new }}=-\frac{\kappa^{2} \alpha}{4 \pi} \int_{0}^{1} d x\left\{\left(1+\frac{x(1-x) M_{Z}^{2}}{M^{2}}\right) \log \left(1-\frac{x(1-x) M_{Z}^{2}}{M^{2}}\right)+\frac{x(1-x) M_{Z}^{2}}{M^{2}}\right\}
$$

and, in the limit $a \equiv\left(M_{Z} / M\right)^{2} \gg 1$, that [79]

$$
\Delta \hat{r}^{\text {new }} \sim-\kappa^{2} \frac{\alpha}{4 \pi}\left(\frac{a}{6} \log a-\frac{a}{9}+O(1)\right) .
$$

With $\Delta \hat{r}^{\text {new }}<0.0010$ at $95 \%$ CL [77], we find, e.g., with $M=m_{e}$, the electron mass, that $|\kappa|<4.1 \cdot 10^{-6}$, whereas if $M=m_{e} / 10$ that $|\kappa|<3.4 \cdot 10^{-6}[19]$.

A variety of astrophysical constraints exist on the magnetic moment of the neutrino, and they can be adapted to our current case as well. They emerge, in particular, from the impact of the additional cooling mechanism such would render on stellar evolution and lifetimes and on supernovae [80, 81. An additional, albeit somewhat weaker, constraint comes from confronting element abundances with the predictions of big-bang nucleosynthesis, to yield, e.g., $\mu_{\nu}<2.9 \cdot 10^{-10} \mu_{B}[82$. These constraints can be significantly weakened by the candidate particle's mass [71]; the ability to produce particles of $\mathcal{O}(10 \mathrm{keV})$ in mass and more in plasma at stellar temperatures is limited. In the case of big-bang nucleosynthesis, the constraints on the magnetic moment of a massive tau neutrino are also weakened, though values as large as $\mu_{\nu} \sim 10^{-6} \mu_{B}$ are nevertheless excluded [82]. 
The constraints we have considered in this section can be weakened by other means as well. Since they arise from the effects of particle production, the most economical mechanism is compositeness; to include this, we need only include a form factor at each electromagnetic vertex. Thus we replace $\kappa \rightarrow \kappa /\left(1-M_{Z}^{2} / M_{c}^{2}\right)^{2}$, where $M_{c}$ is the compositeness scale, in our earlier formulae. Thus for $M=m_{e} / 10$ our earlier bound of $|\kappa|<3.4 \cdot 10^{-6}$ [19] from $\Delta \hat{r}$ relaxes to $|\kappa|<1.5$ if $M_{c}=2 \mathrm{GeV}$. In this scenario, however, the electrically charged constituents may well give rise to other observable effects. One possibility which avoids this would be to give a known charged particle, such as an electron, a small hidden sector interaction, so that it can help constitute dark matter, though its contribution to $\Delta \hat{r}$ has already been taken into account. We proceed to consider the manner in which direct constraints can be set on $\mu$.

\section{GYROMAGNETIC FARADAY EFFECT}

A medium of free electric charges in an external magnetic field is circularly birefringent and gives rise to a Faraday effect [83], as long as the light doe not propagate at right angles to the magnetic field. This effect has long been used in radio astronomy to study the properties of the interstellar medium [84]. A Faraday effect can also arise in an electrically neutral medium in an external magnetic field, if the constituents carry magnetic moments and if they are aligned by that magnetic field to give the medium some net magnetization [20, 21]. We consider the latter possibility exclusively.

To derive the gyromagnetic Faraday effect, we apply a magnetic induction $\boldsymbol{B}_{0}$ in a magnetizable medium with circularly polarized electromagnetic waves propagating parallel to it. The external field induces a magnetization $\boldsymbol{\mathcal { M }}_{\text {tot }}$, i.e., a net magnetic moment/volume, where $\mathcal{M}_{\text {tot }}=\mathcal{M}_{0}+\mathcal{M}$ and $\mathcal{M}_{0}$ results from $\boldsymbol{B}_{0}$ alone. The total magnetization of a medium at rest in the laboratory frame obeys the Larmor precession formula

$$
\frac{d \mathcal{M}_{\text {tot }}}{d t}=\frac{g \mu_{M}}{\hbar} \mathcal{M}_{\text {tot }} \times \boldsymbol{B}_{\text {tot }}
$$

so that $g \mu_{M} / \hbar$, noting $\mu_{M} \equiv e \hbar / 2 M$ with $e>0$ for a particle of mass $M$, is the gyromagnetic ratio of the magnetic-moment-carrying particle. We note $\mu=S g \mu_{M}$, where $S$ is the spin of the particle. The gyromagnetic Faraday effect was first derived for a ferromagnetic material [20, 21], for which use of the magnetic field $\boldsymbol{H}$ is appropriate. Since dark matter is only weakly self-interacting at most, our hypothesized dark matter should be treated as a paramagnetic material — so that we employ the magnetic induction $\mathbf{B}$ throughout, though the use of $\boldsymbol{H}$ is also commonplace [85]. Corrections to the Larmor formula result if the medium's particles move at a significant fraction of the speed of light, or if the particles possess a non-zero electric dipole moment [86]. We shall neglect the latter possibility and, moreover, shall consider dark-matter candidates for which relativistic effects are ultimately small corrections.

To determine the relativistic corrections to Eq. (6), we first construct the covariant classical equation of motion for a single spin in homogeneous electromagnetic fields. This is germane as we can and indeed do neglect the mutual interactions of the dark-matter particles, so that the magnetization is given by the quantum-mechanical expectation value of the spin operator for a single particle times the number density; and the time evolution of the expectation value is itself described by that of the associated classical equation of motion. The latter, for a charged particle with a spin, is given by the Bargmann-Michel-Telegdi 
equation [86]. We cannot use this result directly because no forces act on electrically neutral particles in homogeneous electromagnetic fields, so that no Thomas precession term is present [87]. Nevertheless, well-known treatments [83] can be readily adapted to this case. Requiring $d \mathcal{U}^{\alpha} / d \tau=0$, where $\mathcal{U}=c \gamma(1, \boldsymbol{\beta})$ is the 4-velocity of the particle in the laboratory frame and $\mathcal{S}$ is its spin, namely $\mathcal{S}=\left(\mathcal{S}^{0}, \mathcal{S}\right)$, we find that

$$
\frac{d \mathcal{S}^{\alpha}}{d \tau}=\frac{g \mu_{M}}{\hbar}\left(F^{\alpha \beta} \mathcal{S}_{\beta}+\frac{U^{\alpha}}{c^{2}}\left(\mathcal{S}_{\lambda} F^{\lambda \mu} U_{\mu}\right)\right),
$$

where $F^{\alpha \beta}$ is the field-strength tensor in SI units and $\tau$ is the proper time of the particle. Thus in the laboratory frame the magnetization evolves as

$$
\gamma \frac{d \mathcal{M}_{\mathrm{tot}}}{d t}=\frac{g \mu_{M}}{\hbar}\left(\boldsymbol{\mathcal { M }}_{\mathrm{tot}} \times\left(\boldsymbol{B}_{\mathrm{tot}}-\boldsymbol{\beta} \times \frac{\boldsymbol{E}_{\mathrm{tot}}}{c}\right)+\gamma^{2} \boldsymbol{\beta}\left(\boldsymbol{\beta} \times \mathcal{M}_{\mathrm{tot}}\right) \cdot\left(\boldsymbol{B}_{\mathrm{tot}}-\boldsymbol{\beta} \times \frac{\boldsymbol{E}_{\mathrm{tot}}}{c}\right)\right),
$$

where we emphasize $\gamma$ is the Lorentz factor, namely $\gamma \equiv 1 / \sqrt{1-\beta^{2}}$. To proceed, we separate $\boldsymbol{B}_{\text {tot }}$ and $\boldsymbol{E}_{\text {tot }}$ as $\boldsymbol{B}_{\text {tot }}=\boldsymbol{B}_{0}+\boldsymbol{B}$ and $\boldsymbol{E}_{\text {tot }}=\boldsymbol{E}_{0}+\boldsymbol{E}$, so that $\boldsymbol{M}_{0}$ results exclusively from the external electromagnetic fields $\boldsymbol{B}_{0}$ and $\boldsymbol{E}_{0}$. Working to leading order in the small quantities $\mathcal{M}, \boldsymbol{B}$, and $\boldsymbol{E}$, which arise in the presence of electromagnetic radiation, we have

$$
\begin{aligned}
\gamma \frac{d \mathcal{M}}{d t}= & \frac{g \mu_{M}}{\hbar}\left(\mathcal{M}_{0} \times\left(\boldsymbol{B}_{0}-\boldsymbol{\beta} \times \frac{\boldsymbol{E}_{0}}{c}\right)+\mathcal{M} \times\left(\boldsymbol{B}_{0}-\boldsymbol{\beta} \times \frac{\boldsymbol{E}_{0}}{c}\right)+\mathcal{M}_{0} \times\left(\boldsymbol{B}-\boldsymbol{\beta} \times \frac{\boldsymbol{E}}{c}\right)\right. \\
& \left.+\gamma^{2} \boldsymbol{\beta}\left(\left(\boldsymbol{\beta} \times \mathcal{M}_{0}\right) \cdot\left(\boldsymbol{B}_{\mathrm{tot}}-\boldsymbol{\beta} \times \frac{\boldsymbol{E}_{\mathrm{tot}}}{c}\right)+(\boldsymbol{\beta} \times \boldsymbol{\mathcal { M }}) \cdot\left(\boldsymbol{B}_{0}-\boldsymbol{\beta} \times \frac{\boldsymbol{E}_{0}}{c}\right)\right)\right) .
\end{aligned}
$$

We can consider the evolution of the dark matter magnetization in vacuum, i.e., in the absence of ordinary matter, or in matter. Since the largest external fields we can apply obey $B_{0} \gg E_{0} / c$ in vacuum, we set $E_{0}=0$ henceforth. We note, however, that the atomic-scale separation of electric charges in matter permit the opposite limit, $B_{0} \ll E_{0} / c$, so that the analysis of the magnetization in that case can be altogether distinct. We set this possibility aside for later discussion and continue with the analysis in vacuum. We note that the ability to establish a vacuum relies on the presence of matter with conventional electromagnetic and strong couplings; dark matter is sufficiently weakly interacting that vacuum technology does not affect it. Hence we use "vacuum" to connote the absence of ordinary matter. In this case we apply $\boldsymbol{B}_{0}$ in the $\hat{\boldsymbol{x}}$-direction and choose $\boldsymbol{\beta}$ to be parallel or antiparallel to $\hat{\boldsymbol{x}}$ as well. As we have mentioned, the entry of dark matter into the magnetic field region acts as a spin filter device. The dark matter which does enter the apparatus can thus possess a net magnetization, so that $\mathcal{M}_{0}$ is in the $\hat{\boldsymbol{x}}$-direction ${ }^{1}$. As a result Eq. (9) reduces to

$$
\gamma \frac{d \boldsymbol{M}}{d t}=\frac{g \mu_{M}}{\hbar}\left(\boldsymbol{M} \times \boldsymbol{B}_{0}+\mathcal{M}_{0} \times\left(\boldsymbol{B}-\boldsymbol{\beta} \times \frac{\boldsymbol{E}}{c}\right)\right) .
$$

Choosing the wave vector $\boldsymbol{k}$ of the light in the $\hat{\boldsymbol{x}}$-direction as well, we recall $\boldsymbol{E}=-c \hat{\boldsymbol{x}} \times \boldsymbol{B}$ and let $\boldsymbol{B}(\boldsymbol{x}, t)=B_{ \pm} \boldsymbol{e}_{ \pm} \exp \left(i k_{ \pm} x-i \omega t\right)$, where $\boldsymbol{e}_{ \pm} \equiv \hat{\boldsymbol{y}} \pm i \hat{\boldsymbol{z}}$. We define the polarization

\footnotetext{
1 This follows irrespective of the sign of $g$. For $g<0$, however, the spins preferentially point in the $\pm \hat{\boldsymbol{x}}$ direction.
} 
state with positive helicity, $\mathbf{e}_{+}$, to be right-handed, which differs from the convention used in optics. In steady state, we find $\mathcal{M}=\mathcal{M}_{ \pm} \boldsymbol{e}_{ \pm}$and finally that

$$
\mathcal{M}_{ \pm}= \pm \frac{\omega_{M}(1+\beta)}{\gamma \omega \pm \omega_{B}} B_{ \pm} \equiv \chi_{ \pm} B_{ \pm}
$$

where we have chosen $\boldsymbol{\beta}=-\beta \hat{\boldsymbol{x}}$ and defined $\omega_{M} \equiv g \mu_{M} \mathcal{M}_{0} / \hbar$ and $\omega_{B} \equiv g \mu_{M} B_{0} / \hbar$. Since $k_{ \pm}=(\omega / c) \sqrt{1+\chi_{ \pm}}$, we have

$$
k_{ \pm}=\frac{\omega}{c} \sqrt{1 \pm \frac{\omega_{M}(1+\beta)}{\gamma \omega \pm \omega_{B}}}
$$

or that

$$
k_{+}-k_{-}=\frac{\omega_{M}(1+\beta)}{\gamma c}\left(1+\mathcal{O}\left(\frac{\omega_{B}^{2}}{\gamma^{2} \omega^{2}}\right)\right)
$$

where we note for conceivable light sources that $\omega \gg \omega_{B}, \omega_{M}$. Physically, the magnetic field associated with the passing electromagnetic wave tugs on the spinning particle in a direction perpendicular to $\mathcal{M}_{0}$, prompting it to emit radiation which interferes with the light traveling in the forward direction, generating the birefringence. The Faraday rotation angle $\phi$ is simply $\phi=\left(k_{+}-k_{-}\right) l / 2$, where $l$ is the total distance travelled by the photon. The quantity $\omega_{M}$ is signed, so that the sense of the rotation angle determines the sign of $g$. If $\beta=0$, we recover the result of Ref. [19], whereas in the extreme relativistic limit, i.e., as $\gamma \rightarrow \infty, \chi_{ \pm} \rightarrow 0$ and thus $\phi$ vanishes as well. The average value of $k_{ \pm}$is not altered to leading order in small quantities, namely,

$$
k_{\text {avg }} \equiv \frac{1}{2}\left(k_{+}+k_{-}\right)=\frac{\omega}{c}\left(1+\mathcal{O}\left(\frac{\omega_{B} \omega_{M}}{\gamma^{2} \omega^{2}}\right)\right),
$$

so that an appreciable Faraday rotation can accrue in the absence of an effect on the average group velocity. Thus far we have considered the Faraday rotation of linearly polarized light consequent to passage a distance $l$ through a medium; practical considerations demand that we determine its properties under reflection as well. If we reverse the direction of the light, Eq. (11) is unaltered save for the sign of the term in $\beta$. The last does change sign since $\boldsymbol{E}=-c \hat{\boldsymbol{k}} \times \boldsymbol{B}$. Thus if we set $\beta=0$, an initially right-handed circularly polarized wave, e.g., travels both forward and backward with wave number $k_{+}$, so that the rotation angle accrues coherently under momentum reversal. The additional Faraday rotation associated with the explicit $\beta$-dependent term in Eq. (12), however, cancels under a round-trip transit. Such contrasting behavior is long familiar from the study of birefringence in chiral media [22, which break macroscopic parity invariance. Similar conclusions have been drawn from an analysis of parity-violating photon-external-field interactions as well [88]. Thus the net rotation angle after a round-trip, or after many, of a total travel length $l$ is

$$
\phi_{0}=\frac{\omega_{M} l}{2 \gamma c}\left(1+\mathcal{O}\left(\frac{\omega_{B}^{2}}{\gamma^{2} \omega^{2}}\right)\right) .
$$

Neglecting the $\mathcal{O}\left(\omega^{-2}\right)$ corrections and working in the $\beta \rightarrow 0$ limit, this becomes simply

$$
\phi_{0}=\frac{g \mu_{M} \mathcal{M}_{0} l}{2 \hbar c}\left(1+\mathcal{O}\left(\beta^{2}\right)\right)
$$


which agrees with the result of the non-relativistic treatment in Ref. [19]. For a system at rest in thermal equilibrium, the magnetization $\mathcal{M}_{0}$ is a simple function of the applied magnetic field. We recall that for a system of spin $1 / 2$ particles, e.g., each with magnetic moment $\mu$, the magnetization for a system with number density $n_{M}$ at temperature $T$ is [89]

$$
\mathcal{M}_{0}=n_{M} \mu \tanh \left(\frac{\mu B_{0}}{k_{B} T}\right) \text {, }
$$

though it is of little practical relevance to the current circumstance, for the system we consider approaches thermal equilibrium extremely slowly. That is, a dilute gas in an external magnetic field polarizes through spontaneous emission, and the rate $W$ for this process is given by that of a magnetic dipole transition [90]:

$$
W=\frac{4}{3 \hbar}\left(\frac{\omega}{c}\right)^{3}\left(g \mu_{M}\right)^{2}
$$

where $\omega=g \mu_{M} B / \hbar$. Thus even if $\mu_{M}=\mu_{B}$, the Bohr magneton, and $B=25 \mathrm{~T}$, we would have $W \sim 1 \cdot 10^{-6} \mathrm{~s}^{-1}$, which is trivial compared to the average rate with which dark matter is expected to transit an experimental apparatus. Although dark matter may possess some primordial magnetization, it is likely so small [19] that it is important to realize other means of polarizing it. For the particular geometry we consider, as we have noted, the onset of the magnetic field region acts as a spin filter device. Although this method should yield some net magnetization for any non-zero spin $S$, we explicitly assume a spin $1 / 2$ candidate in what follows. If the velocity $\boldsymbol{v}_{M}$ of the incoming particles is aligned with the direction of the magnetic field, then particles with $v_{M} \equiv \beta c<v_{\text {stop }}$ can only enter the magnetic field region if their magnetic moment is aligned with it, where $v_{\text {stop }}$ is such that

$$
\frac{1}{2} M v_{\text {stop }}^{2}=|\mu| B_{0}
$$

We have set any external electric field to zero and have neglected corrections of $\mathcal{O}\left(\beta^{2}\right)$. The dark matter which does enter the apparatus can thus possess a net magnetization; namely,

$$
\mathcal{M}_{0}=n_{M} \mu \mathcal{P}
$$

where $\mathcal{P}$ is the polarization of the spins. We define $\mathcal{P} \equiv\left(N_{+}-N_{-}\right) /\left(N_{+}+N_{-}\right)$, where $N_{+}$ and $N_{-}$are the number of spins pointing in and against the direction of $\boldsymbol{B}_{0}$, respectively. If $\mu \rightarrow-\mu$ then $\mathcal{M}_{0} \rightarrow \mathcal{M}_{0}$ just as in Eq. (17). We study the value of $\mathcal{P}$ as a function $\mu B_{0}$, $M$, and astrophysical parameters in the next section.

Before proceeding, we return to the notion of studying the Faraday rotation of dark matter passing through ordinary matter. In this regard, we wish to consider matter comprised of atoms with closed electron shells, so that there are no unpaired electrons present to engender a gyromagnetic Faraday effect. The exceptionally large electric fields associated with atoms and nuclei [91 ${ }^{2}$, make it possible for $\left|\boldsymbol{\beta} \times \boldsymbol{E}_{0}\right| / c$ to exceed presently achievable external magnetic fields [92]. Such considerations yield significant limits on the neutron electric dipole moment [93], e.g., from neutron-noble gas scattering [91, 94]. Returning to Eq. (9), we choose $\hat{\boldsymbol{x}} \| \hat{\boldsymbol{k}}$ as in previous case, but now choose $\boldsymbol{\beta} \perp \hat{\boldsymbol{x}}$ so that $\boldsymbol{\beta} \times \boldsymbol{E}_{0}$ can also be in

\footnotetext{
${ }^{2} \mathrm{In} \mathrm{H}$-atom, e.g., a test charge a Bohr radius away from the proton sees $E / c \approx 2 \cdot 10^{3} \mathrm{~T}$.
} 
the $\hat{\boldsymbol{x}}$ direction. Counting $\left|\boldsymbol{\beta} \times \boldsymbol{E}_{0}\right| / c$ as a parameter of $\mathcal{O}(1)$ and neglecting terms of $\mathcal{O}\left(\beta^{2}\right)$ and higher, we have

$$
\frac{d \mathcal{M}}{d t}=\frac{g \mu_{M}}{\hbar}\left(-\mathcal{M} \times\left(\boldsymbol{\beta} \times \frac{\boldsymbol{E}_{0}}{c}\right)+\mathcal{M}_{0} \times\left(\boldsymbol{B}-\boldsymbol{\beta} \times \frac{\boldsymbol{E}}{c}\right)\right),
$$

and the steady-state solution

$$
\mathcal{M}_{ \pm}= \pm \frac{\omega_{M}}{\omega \mp \omega_{E}} B_{ \pm}
$$

where $\omega_{E} \equiv g \mu_{M}\left|\boldsymbol{\beta} \times \boldsymbol{E}_{0}\right| / \hbar c$, which yields the rotation angle

$$
\phi_{0}=\frac{g \mu_{M} \mathcal{M}_{0} l}{2 \hbar c}\left(1+\mathcal{O}\left(\frac{\omega_{E}^{2}}{\omega^{2}}, \beta^{2}\right)\right),
$$

irrespective of whether round-trip paths are executed by the light. In this context, then, the effective magnetic field is relevant simply to the value of $\mathcal{M}_{0}$. Here, too, we need to determine the polarization of the dark matter which penetrates the material. Suppose $\boldsymbol{\beta}=\beta \hat{\boldsymbol{y}}$ and that it is possible to choose a material for which $E_{0 z} \gg E_{0 x}$, so that the effective magnetic field is in the $\hat{\boldsymbol{x}}$ direction. The force on the dark-matter particle in entering the medium is $\boldsymbol{\nabla}\left(\boldsymbol{\mu} \cdot\left(\boldsymbol{\beta} \times \boldsymbol{E}_{0} / c\right)\right)$; since we can expect the magnitude of $\boldsymbol{E}_{0}$ to depend on $y$, a longitudinal Stern-Gerlach effect is still possible in this geometry. A force in the $z$ direction can engender the more familiar transverse Stern-Gerlach effect, but the increasing diameter of the laser beam as a result of scattering in its passage through the material may make it impossible to exploit this feature. Thus we tentatively conclude that it ought be possible to realize a meaningful Faraday rotation study in matter as well, with potential gains in sensitivity to a possible dark matter magnetic moment. We now return to the vacuum case, to describe how such an experiment can be realized and to estimate the limits on $\mu$ one could possibly obtain.

\section{A FARADAY ROTATION EXPERIMENT}

Although the Faraday rotation effect we discuss can be found through correlation studies of the polarization of the cosmic microwave background [19], a terrestrial Faraday rotation experiment offers a number of advantages. Current bounds on primordial magnetic fields [39] make any primordial magnetization associated with dark matter small [19] and difficult to probe, particularly in experiments executed over terrestrial length scales. However, in this case, as we shall demonstrate, one can apply a very strong magnetic field of known strength, and polarize the dark matter to an appreciable degree. Moreover, as we have seen, the Faraday rotation associated with a magnetic moment accrues coherently under momentum reversal, so that the measurement can be made in a small cavity and yet have a long effective path length. Measurements of very small rotation angles are also possible; the recent PVLAS experiment, for example, was able to achieve sensitivity to rotation angles of $\mathcal{O}\left(10^{-8}\right)[36$ - this stands in constrast to a sensitivity of $\mathcal{O}\left(10^{-4}\right)$ anticipated with the future CMBpol satellite [95].

A schematic of the Faraday rotation experiment we propose is illustrated in Fig. 1. Its ingredients comprise a laser, a linear polarizer, an evacuated optical cavity through which the light makes multiple passes and to which a longitudinal, steady magnetic field has been applied, and an analyzer. The technical requirements of a sensitive Faraday rotation 


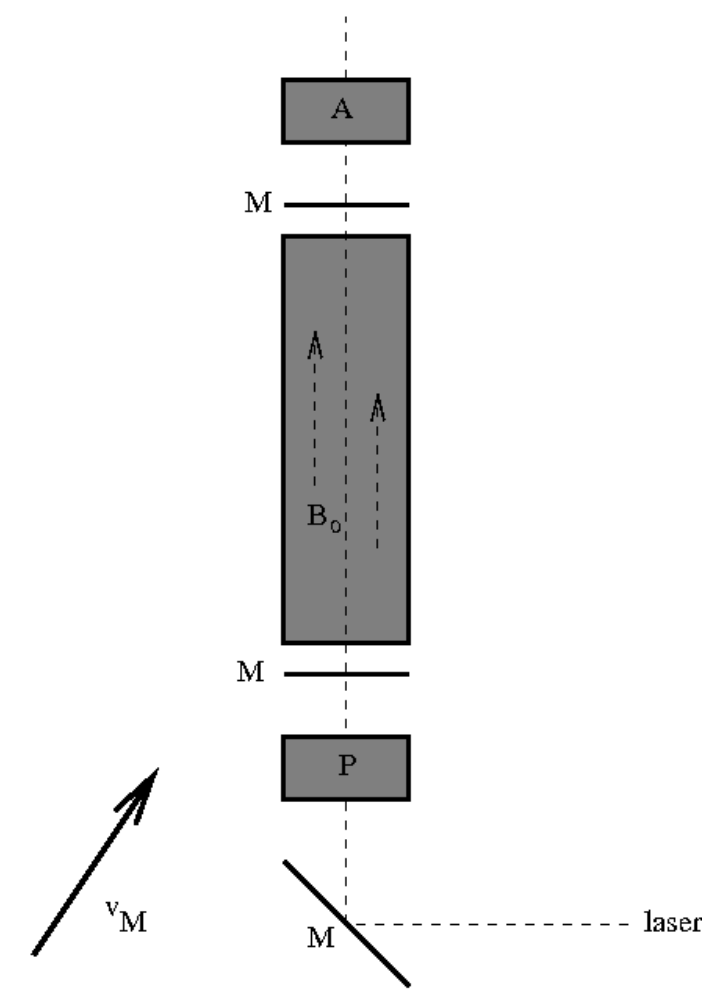

FIG. 1: Schematic of the Faraday rotation experiment described in text.

measurement may demand a more sophisticated setup; however, such details are not needed for our estimate of the limit on $\mu$ for a given sensitivity to the rotation angle. We emphasize that since dark matter carries neither electric charge nor suffers strong interactions [9], it is unaffected by vacuum pumps and, indeed, can pass into cavities free from ordinary matter. The generic setup we propose, save for the nature of the magnetic field, is common to the PVLAS experiment [36], which investigated the optical properties of the vacuum, as well. As that experiment was sensitive to extremely small changes in the photon polarization, we adopt it as a reference - we use certain of the parameters chosen in that experiment in order to estimate the achievable bounds on the magnetic moment as a function of the mass of the dark-matter candidate. A remark concerning the orientation of the apparatus in our schematic is in order. In our derivation of Eq. (16) we chose $\hat{\boldsymbol{\beta}}=\hat{\boldsymbol{x}}$, though our result is of more general validity. That is, if we return to Eq. (9), setting $\boldsymbol{E}_{0}=0$ and working to $\mathcal{O}\left(\beta^{2}\right)$, we see that the absence of the $\mathcal{O}(\beta)$ term in Eq. (16) results from the transformation properties of $\boldsymbol{E}$ under momentum reversal - regardless of the direction of $\boldsymbol{\beta}$. Consequently, we need not orient the direction of the magnetic field with respect to the dark matter "wind" in any particular way, as we indicate in Fig. 1. It will turn out, however, that the choice of the orientation of the apparatus with respect to the Earth's velocity can modify the efficacy of the longitudinal Stern-Gerlach device.

In order to evaluate the sensitivity of the scheme we suggest to a dark matter magnetic moment, we need to make assumptions concerning its local velocity distribution and number density. Both of the latter quantities are relevant to the computation of $\mathcal{M}_{0}$ via Eq. (20), which gives rise to the rotation angle in Eq. (16). To do this we adopt the "canonical model" [96, 97] employed in the analysis of direct detection experiments [24, 25, 26] for cold dark matter. That is, we assume that the dark matter in our galaxy resides in a non-rotating 
halo and that the velocity distribution function $f(\boldsymbol{v})$ in that halo is that of an isothermal sphere. This assumption is motivated by simplicity. More realistic distributions can affect the expected event rates, as well as their temporal variation [98, 99, 100]. We note that $\boldsymbol{v}=\boldsymbol{v}_{M}+\boldsymbol{v}_{E}$, where $\boldsymbol{v}_{M}$ is the velocity of dark matter relative to the Earth, which we introduced earlier, and $\boldsymbol{v}_{E}$ is the velocity of the Earth relative to the nonrotating halo of the galaxy. The value of $v$ can range up to the galactic escape velocity, roughly $650 \mathrm{~km} / \mathrm{s}$ [96, though we do not impose this cut-off in what follows, because the normalization of the resulting distribution differs by less than $1 \%$ [97]. Thus the form of $f$ is that of a MaxwellBoltzmann distribution:

$$
f\left(\boldsymbol{v}_{M}, \boldsymbol{v}_{E}\right)=\frac{1}{\pi^{3 / 2} v_{0}^{3}} \exp \left(-\frac{\left(\boldsymbol{v}_{M}+\boldsymbol{v}_{E}\right)^{2}}{v_{0}^{2}}\right) .
$$

The velocity $v_{0}$ is related to the root-mean-square velocity of the distribution; for a galaxy with a flat rotation curve it is argued to be equal to the radial velocity of the galactic disk [101] and thus in practice is taken to be $v_{0} \approx 220 \mathrm{~km} / \mathrm{s} \mathrm{[96].} \mathrm{The} \mathrm{velocity} \boldsymbol{v}_{E}$ is determined by the sum of

$$
\boldsymbol{v}_{E}=\boldsymbol{u}_{r}+\boldsymbol{u}_{s}+\boldsymbol{u}_{e},
$$

where $\boldsymbol{u}_{r}$ is the velocity of the galactic disk in an inertial reference frame, $\boldsymbol{u}_{s}$ is the velocity of the Sun with respect to the galactic disk, and $\boldsymbol{u}_{e}$ is the velocity of the Earth about the Sun. Assuming the Milky Way is axisymmetric, $\boldsymbol{u}_{r}$ is fixed by the circular velocity at the Sun's radius from the galactic center, which is $220 \pm 20 \mathrm{~km} / \mathrm{s}$ [102, 103]. In galactic coordinates $(-\hat{\boldsymbol{r}}, \hat{\boldsymbol{l}}, \hat{\boldsymbol{z}})$ [104], in $\mathrm{km} / \mathrm{s}$, we thus employ [96]: $\boldsymbol{u}_{r}=(0,220,0)$, which follows if the Milky Way is axisymmetric, and $\boldsymbol{u}_{s}=(9,12,7)$. The approximate speed of the Earth about the Sun is $30 \mathrm{~km} / \mathrm{s}$, whereas its approximate speed about its axis is $0.5 \mathrm{~km} / \mathrm{s}$. We ignore the effects of the Earth's rotation about its axis in our analysis, as it is no larger than the error in $\boldsymbol{u}_{s}$ [104, 105]. Moreover, $\boldsymbol{v}_{E}$ is dominated by motion along the longitudinal coordinate $\hat{\imath}$. Noting this and that the ecliptic is oriented at an angle of roughly $60^{\circ}$ with respect to the galactic equatorial plane means we can approximate the piece of $\boldsymbol{u}_{E}$ in the longitudinal direction $\hat{\boldsymbol{l}}$ as $30 \cos 60^{\circ} \cos (2 \pi((t-152.5) / 365.25))$, to estimate $\boldsymbol{v}_{E} \approx(232+15 \cos (2 \pi((t-$ $152.5) / 365.25))) \hat{\boldsymbol{l}}[96$. We retain this approximation for simplicity, though the presence of the other components of $\boldsymbol{v}_{E}$, as well as a more realistic velocity distribution, are important to an assessment of the event rates, the size and phase of any temporal variations therein, and dark-matter exclusion limits to better than $\mathcal{O}(100 \%)$ [98, 99, 106. We note, too, that more precise determinations of the astronomical inputs also exist [104, 105].

Finally, to complete our description of the model, we choose a dark matter mass density of $\rho=0.3 \mathrm{GeV} / \mathrm{cm}^{3}$ [96]. The currently accepted range for $\rho$ is $0.2-0.4 \mathrm{GeV} / \mathrm{cm}^{3}[107$, 108] for a smooth matter distribution with a spherical halo. Models of galaxy formation which relax the smoothness assumption can give rise to local dark matter densities both larger and smaller than this range [109], where we refer to Ref. [110] for a succinct summary of the possibilities. Some of the uncertainties in the model we outline can be correlated. For example, an elliptical halo, and concomitant triaxial velocity distribution, can yield somewhat higher local densities [111, 112]. Unfortunately, direct information on the darkmatter mass density in our solar system is sparse, and observational bounds exceed the estimate we employ by orders of magnitude [113, 114, 115, 116, 117, 118].

To realize a limit on $\mu$, we assert in what follows that dark matter is comprised of a single type of spin $1 / 2$ particle with fixed $M$ and $\mu$, though we emphasize that the detection of a signal does not require that the particle have spin $1 / 2$. Our numerical estimate is in two 


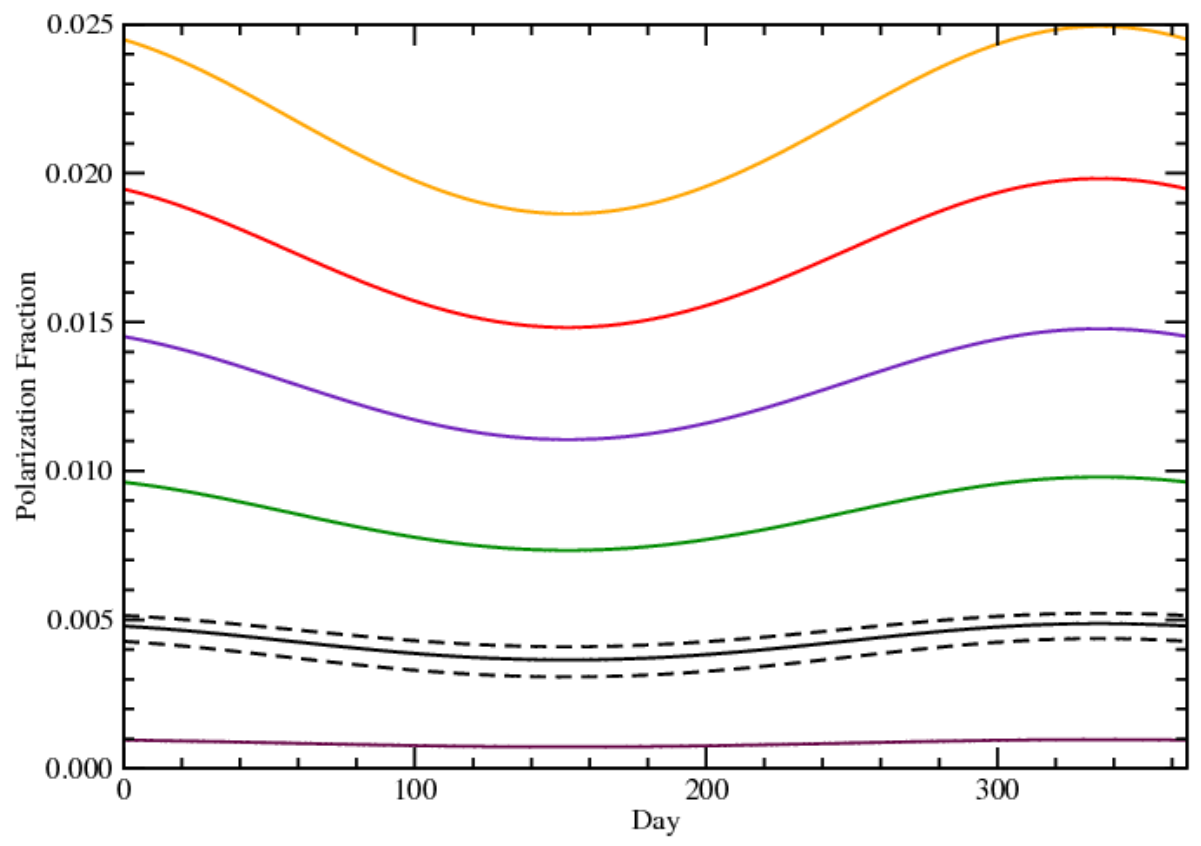

FIG. 2: The polarization fraction $|\mathcal{P}|$ after Eq. (27) plotted as a function of day for various $v_{\text {stop }}$ and $v_{0}$. Here $\boldsymbol{v}_{E}$ has been assumed parallel to $\boldsymbol{B}_{0}$; this yields a geometry-independent lower bound to the polarization, as described in text. The solid lines have $v_{0}=220 \mathrm{~km} / \mathrm{s}$ and $v_{\text {stop }}=1,5,10,15,20$, and $25 \mathrm{~km} / \mathrm{s}$, respectively, as one moves in the direction of increasing $|\mathcal{P}|$. The dashed curves have $v_{\text {stop }}=5 \mathrm{~km} / \mathrm{s}$ and $v_{0}=200 \mathrm{~km} / \mathrm{s}$ and $v_{0}=240 \mathrm{~km} / \mathrm{s}$, below and above, respectively, the solid line with the same $v_{\text {stop }}$.

distinct parts. Operating in the canonical model, we first assess the polarization of the dark matter in the magnetic field region as function of $M$ and $\mu$. With this in hand, we can then determine the limit on $|\mu|$ with $M$ which follows from a given limit on the magnitude of the Faraday rotation angle.

We now proceed to estimate the polarization. We assume that the magnetic field is uniform in some direction $\hat{\boldsymbol{x}}$. If this is realized in a finite volume, with no magnetic field external to it, then a longitudinal Stern-Gerlach effect exists at each field boundary - i.e., the "wrong" spin state suffers a repulsive force at each surface. In what follows we assume a slab geometry and estimate the polarization resulting from crossing a single interface; we assume the magnetic field is perpendicular to the interface. In this case the polarization condition is on $\boldsymbol{v}_{M} \cdot \hat{\boldsymbol{B}}_{0} \leq v_{\text {stop }}$, where $v_{\text {stop }}$ is fixed by Eq. 19). Thus the fraction of particles which enter the magnetic field region with $100 \%$ polarization is

$$
f_{\mathrm{pol}}=\frac{1}{2} \int d^{3} v_{M} f\left(\boldsymbol{v}_{M}, \boldsymbol{v}_{E}\right) \Theta\left(v_{\mathrm{stop}}-\left|\boldsymbol{v}_{M} \cdot \hat{\boldsymbol{B}}_{0}\right|\right),
$$

so that its polarization in the magnetic field region is $\mathcal{P}=(\operatorname{sgn} \mu) f_{\mathrm{pol}} /\left(1-f_{\mathrm{pol}}\right)$, and its number density is $n_{M}=\rho\left(1-f_{\text {pol }}\right) / M$. The evaluation of Eq. (26) yields two pieces, which 
are distinguished by whether $\left|\boldsymbol{v}_{M}\right|$ is larger or smaller than $v_{\text {stop }}$. The term with $\left|\boldsymbol{v}_{M}\right| \leq v_{\text {stop }}$ can be evaluated irrespective of the orientation of the magnetic field region, whereas the term with $\left|\boldsymbol{v}_{M}\right| \geq v_{\text {stop }}$ depends on the value of $\boldsymbol{v}_{E} \cdot \boldsymbol{B}_{0}$. With Eq. (24), we see that $f$ is maximized for $\boldsymbol{v}_{M}$ in the neighborhood of $-\boldsymbol{v}_{E}$, so that if $v_{\text {stop }} \ll v_{E}$, the term with $\left|\boldsymbol{v}_{M}\right| \geq v_{\text {stop }}$ dominates $f_{\text {pol }}$, and the relative orientation of $\boldsymbol{v}_{E}$ and $\boldsymbol{B}_{0}$ becomes important. If, moreover, we make $\boldsymbol{v}_{E} \perp \boldsymbol{B}_{0}$, then we can have both $\boldsymbol{v}_{M}=-\boldsymbol{v}_{E}+\boldsymbol{\delta}$ and $\boldsymbol{v}_{M} \cdot \hat{\boldsymbol{B}}_{0}=\boldsymbol{\delta} \cdot \hat{\boldsymbol{B}}_{0}$ with $\delta$ small — we expect $f_{\text {pol }}$ to be maximized for this geometry. Thus to set a limit on $\mu$ irrespective of the orientation of $\boldsymbol{v}_{E}$ and $\boldsymbol{B}_{0}$, we choose $\boldsymbol{v}_{E} \| \hat{\boldsymbol{B}}_{0}$, as this geometry gives the smallest value of $f_{\text {pol }}$ for fixed $\mu$ and $M$. Ultimately the assessment of the limit on $\mu$ in a real experiment will depend on the geometry and orientation of the magnetic field, but our procedure should bound from above the limit on $\mu$ to be found from a given sensitivity to the Faraday rotation angle per unit length. In this, we implicitly assume that dark matter is described by a single constituent. In this special case we find

$$
f_{\text {pol }}^{\|}=\frac{1}{4}\left(\operatorname{erf}\left(\frac{v_{\text {stop }}-v_{E}}{v_{0}}\right)+\operatorname{erf}\left(\frac{v_{\text {stop }}+v_{E}}{v_{0}}\right)\right) .
$$

Since $\operatorname{erf}(z) \rightarrow 1$ as $z \rightarrow \infty, f_{\text {pol }} \rightarrow 1 / 2$ as $v_{\text {stop }} \rightarrow \infty$, as required. The form of Eq. (27) emerges from a partial cancellation of the contributions from the $v_{M} \leq v_{\text {stop }}$ and $v_{M} \geq v_{\text {stop }}$ regimes, so that we pause to consider whether the inclusion of an escape velocity in this particular case could modify our results. In this event, the normalization of Eq. (26) changes slightly, but negligibly [97], and the integrand accrues a factor of $\Theta\left(v_{\text {esc }}-\left|\boldsymbol{v}_{M}+\boldsymbol{v}_{E}\right|\right)$. For $v_{\text {stop }} \ll v_{E}$, this additional factor does not restrict the region of integration unless $v_{M} \gtrsim 600$ $\mathrm{km} / \mathrm{s}$; finally, we conclude that the continued neglect of $v_{\text {esc }}$ is justified. Solving Eq. (19), we find $v_{\text {stop }} \approx 4.51 \mathrm{~km} / \mathrm{s} \sqrt{\kappa B_{0}[T]}\left(m_{e} / M\right)$, where $B_{0}[T]$ is in tesla and $m_{e}$ is the electron mass. Employing a 7 T magnet, as used in the UCNA experiment [119], though magnets of up to $20 \mathrm{~T}$ are commerically available [120], we find for $M=m_{e}$ and $\kappa=1$, with $\mu=\kappa \mu_{M}$, that $v_{\text {stop }} \approx 6 \mathrm{~km} / \mathrm{s}$. The polarization fraction $|\mathcal{P}|$ as a function of day, using $f_{\text {pol }}$ from Eq. (27), is shown for various $v_{\text {stop }}$ and $v_{0}$ in Fig. 2, Day-by-day variations in the polarization exist since $f_{\text {pol }}$ grows larger as $\left|\boldsymbol{v}_{E}\right|$ decreases. The time variation is more marked for the $\boldsymbol{v}_{E} \| \boldsymbol{B}_{0}$ geometry we have chosen, as the precise value of $\boldsymbol{v}_{E}$ impacts the range of velocities which enter the magnetic field region, and, hence, the polarization. For definitenss, we choose day 335 to set limits on $\mu$. The experiment we consider can be sensitive to both annual and daily signal variations, in principle. This follows from the duration of the photon-dark-matter interrogation time, which, in turn, is set by the size and finesse of the cavity in which the experiment is realized. We note that the total travel length $l$ of the laser light in the PVLAS experiment is $l=4.4 \cdot 10^{6} \mathrm{~cm}$ [36], which corresponds to an interrogation time of $\mathcal{O}(0.1 \mathrm{msec})$. This admits the possibility of using pulsed magnetic fields, which can be much stronger as much as $89 \mathrm{~T}$ [121]. Irrespective of this, studies with the magnetic field on and then off are important to establishing a non-zero signal, if it is present.

We now consider how a limit on the magnetic moment follows from our determined polarization and a given sensitivity to the Faraday rotation angle per unit length. For a spin-1/2 candidate, the rotation angle of Eq. (15) can be written as

$$
\phi_{0}=\mathcal{P} \kappa^{2}\left(\frac{m_{e}}{M}\right)^{3} \mu_{B}^{2} \frac{n_{e} l}{\hbar c} \approx\left(6.84 \cdot 10^{-23} \mathrm{~cm}^{2}\right) n_{e}\left[\mathrm{~cm}^{-3}\right] l[\mathrm{~cm}] \mathcal{P} \kappa^{2}\left(\frac{m_{e}}{M}\right)^{3}
$$

with $n_{e} \equiv \rho\left(1-f_{\mathrm{pol}}\right) / m_{e} c^{2}$ and $\mathcal{P}$ from $f_{\mathrm{pol}}$ as per Eq. (27). We note, too, that if dark matter were a mixture of particle and antiparticles of the same mass that the rotation angle 


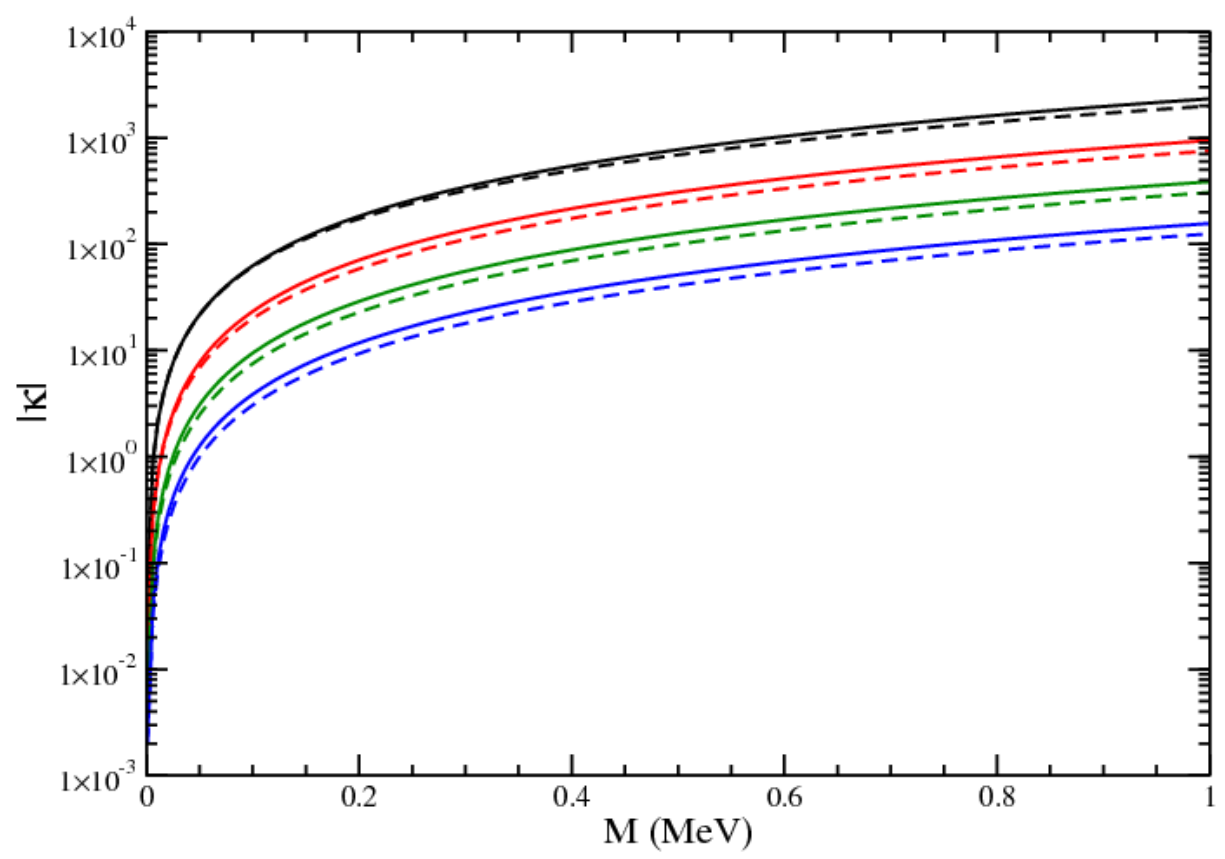

FIG. 3: The limit on $|\kappa|$ at $95 \%$ confidence interval, where $\mu=\kappa \mu_{M}$ and $\mu_{M}=e \hbar / 2 M$, as a function of $M$ up to $1 \mathrm{MeV}$ for various limits on $\phi_{0} / l$ and for different values of the magnetic field $B_{0}$. The solid lines correspond to $B_{0}=7 \mathrm{~T}$, whereas the dashed lines correspond to $B_{0}=20$ $\mathrm{T}$. The limit on $\phi_{0} / l$ in each case is $10^{-12}, 10^{-13}, 10^{-14}$, and $10^{-15} \mathrm{rad} / \mathrm{m}$, respectively, at $95 \%$ confidence interval, as one sweeps from the top to the bottom of the figure.

could cancel, at least in part. We can rewrite Eq. (28) in terms of a limit on $|\mu|$ by replacing $\kappa m_{e} / M$ with $\mu\left[\mu_{B}\right]$. The most stringent limits on $|\kappa|$ emerge for $M \ll m_{e}$. For fixed $M$, its numerical value rests on the ability to determine $\phi_{0} / l$. In the PVLAS experiment [36], the error in $\phi_{0} / l$ is determined to be $0.5 \cdot 10^{-12} \mathrm{rad} / \mathrm{m}$. Since $l=4.4 \cdot 10^{4} \mathrm{~m}, \phi_{0}$ itself is determined to $2.2 \cdot 10^{-8} \mathrm{rad}$. Assuming then that $\phi_{0} / l$ can be determined to $1 \cdot 10^{-12} \mathrm{rad} / \mathrm{m}$ at $95 \%$ confidence interval, we find the limit on $|\kappa|$, or $|\mu|$, as a function of $M$. In Fig. 3 we show the limit on $|\kappa|$ for candidate masses up to $1 \mathrm{MeV}$, whereas in Fig. 4 we show the limit on $|\mu|$ for masses from 1 to $100 \mathrm{MeV}$. The limits depend on the dark-matter polarization $\mathcal{P}$ as well. To illustrate the relative importance of $\mathcal{P}$ and the determination of $\phi_{0} / l$ to the limit on $|\kappa|$, we show not only how the limits change if $B_{0}$ is increased from 7 to $20 \mathrm{~T}$ but also, in Fig. 5, the value of $|\mathcal{P}|$ associated with each limiting value of $|\kappa|$ in Fig. 3. The increase in $B_{0}$ makes little difference at the lightest mass scales we consider, simply because the polarization at these scales is already near unity. Indeed, increasing the value of $B_{0}$ simply increases the largest value of $M$ for which we can reasonably constrain the value of $|\kappa|$. Our assessment of the polarization as per Eq. (27) is that of a lower bound, yet the polarizations we find are large enough that our upper bounds on $|\kappa|$ are no more than a factor of a few larger than what we would find after a realistic simulation of the geometry and orientation of the magnetic field region. It thus emerges that the limits to be set depend 


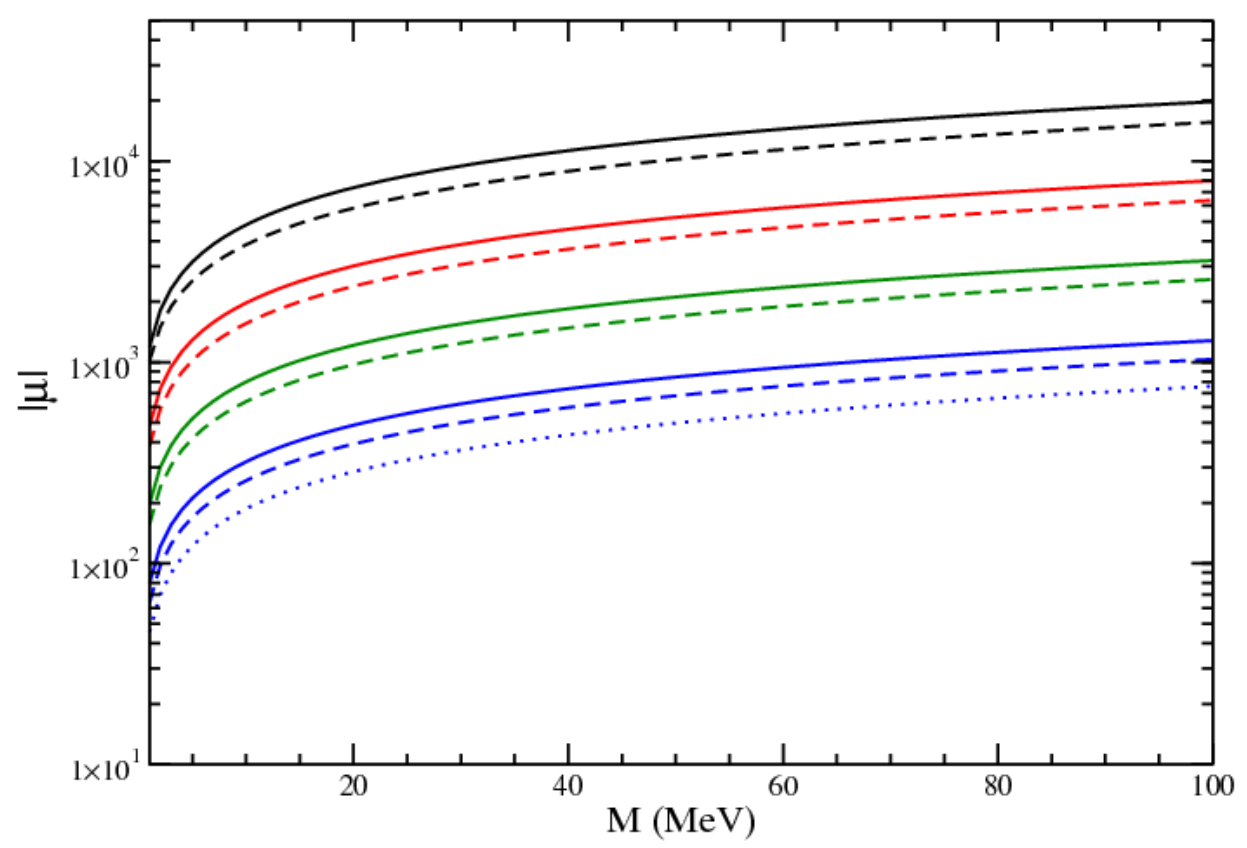

FIG. 4: The limit on $|\mu|$ in units of $\mu_{B}$ at $95 \%$ confidence interval, as a function of $M$ for masses ranging from 1 to $100 \mathrm{MeV}$ for various limits on $\phi_{0} / l$ and for different values of the magnetic field $B_{0}$. We employ the notation of Fig. 3 throughout, and note that dotted line corresponds to $B_{0}=89 \mathrm{~T}$ and a limit on $\phi_{0} / l$ of $10^{-15} \mathrm{rad} / \mathrm{m}$ at $95 \%$ confidence interval.

overwhelmingly on the ability to determine $\phi_{0} / l$. Let us consider then the determination of this quantity and its consequences carefully.

In Figs. (3) and (4) we show how the limits improve as the determination of $\phi_{0} / l$ improves by orders of magnitude. This can be realized by either increasing $l$ or by bettering the measurement of the rotation angle. We note, in particular, that better determinations of $\phi_{0}$ are possible [122, and indeed that precision polarimetry at the shot-noise limit has been demonstrated [123, 124]. In this limit the error in $\phi_{0}$ is set, crudely, by the number of photons counted, $\delta \phi_{0} \simeq 1 /\left(2 \sqrt{I_{o} T}\right)$, where $I_{0}$ is the number of photons per second and $T$ is the measurement time. Assuming a $1 \mathrm{~W}$ laser in the optical regime, so that $E_{\gamma} \approx 1 \mathrm{eV}$, we have $\delta \phi_{0} \simeq 2 \cdot 10^{-10} \mathrm{rad}-\mathrm{s} / \sqrt{T[s]}[122,124$. Moreover, it has been demonstated that the use of squeezed light makes it possible to evade this quantum limit and realize yet more precise polarimetry [124, 125]. All this suggests that the significant gains in the determination of $\phi_{0} / l$ we consider and more are indeed possible. Interestingly, as the determination of $\phi_{0} / l$ becomes more precise, increasing the value of $B_{0}$ becomes more important to an improved limit on $|\kappa|$. We note, e.g., that if $M=100 \mathrm{MeV}$ and $B=89 \mathrm{~T}$ that the polarization fraction associated with the value of $|\mu|$ which follows from a limit of $\phi_{0} / l$ of $10^{-15} \mathrm{rad} / \mathrm{m}$ at $95 \%$ confidence level is $|\mathcal{P}| \approx 0.09$.

We have determined the direct limits on $|\mu|$, or $|\kappa|$, which would follow from a nonobservation of Faraday rotation at a given sensitivity. As they stand the limits constrain 


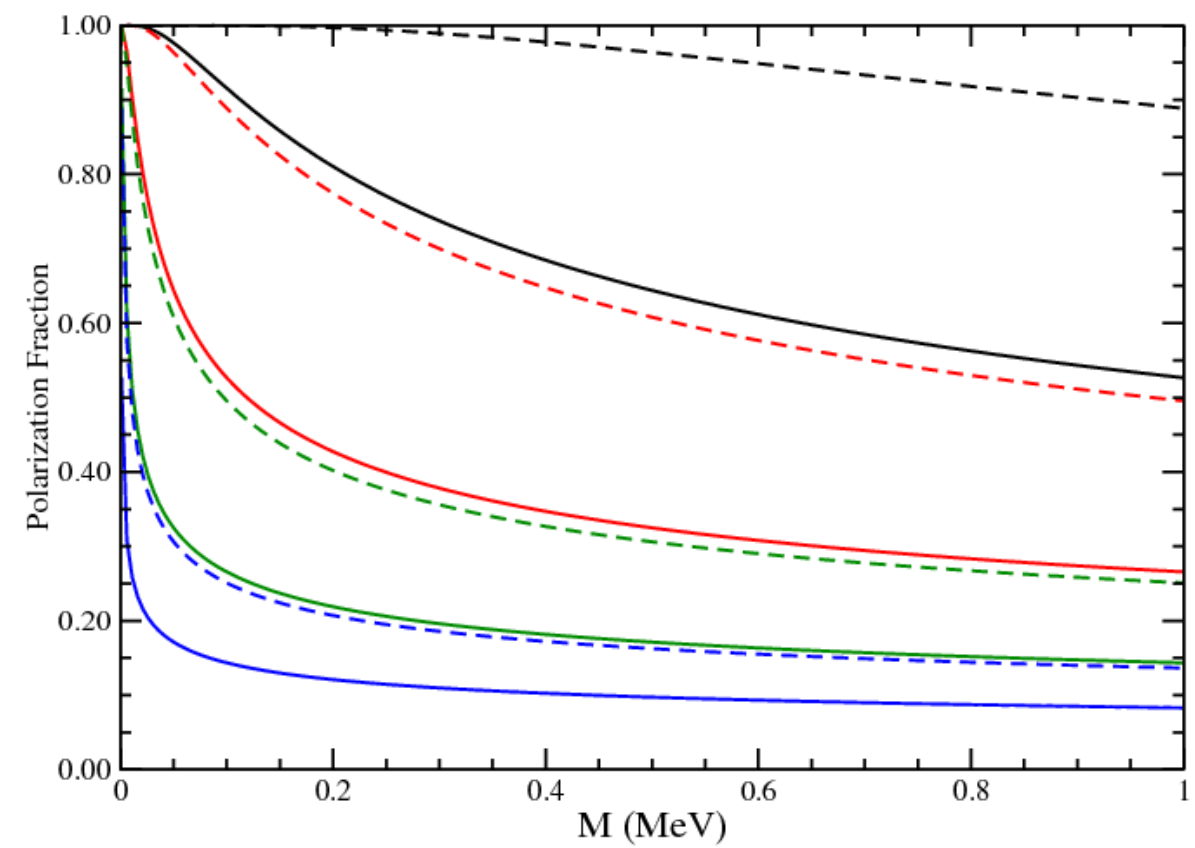

FIG. 5: The assessed polarization fraction $|\mathcal{P}|$ associated with the limiting value of $|\kappa|$ for fixed $M$ shown in Fig. 3. We assume, as in Fig. 2, that $\boldsymbol{v}_{E} \| \boldsymbol{B}_{0}$, to yield a geometry-independent lower bound to the polarization. The conditions which specify the various curves are as given in Fig. 3 .

the possibility that the dark-matter particle is a composite built from electromagnetically charged constituents. Significantly more severe limits would be needed to challenge the possibility that a dark-magnetic magnetic moment exists by dint of quantum-loop effects. Thus we wish to consider the prospect of radical improvement in our limits. Improvements can come from bettering the determination of $\phi_{0} / l$, increasing $B_{0}$, or, finally, noting Eq. (28), from increasing the number density $n_{e}$. The ability to limit the value of $\phi_{0} / l$ beyond that established by the PVLAS experiment [36] has already been demonstrated [123, 124], and the ultimate limit to the determination of $\phi_{0} / l$ has not yet been established [125]. Moreover, as discussed in Sec. IV] mounting the Faraday rotation experiment in matter can yield magnetic fields which are larger by orders of magnitude, and can possibly realize larger values of the dark-matter polarization $\mathcal{P}$ at fixed $\mu$ and $M$. Figures 3 and 5 give a sense of the outcome as a result of such improvements. Let us now turn to the last possibility.

For the usually assumed Galactic halo density, $n_{e}$ is only some $600 \mathrm{~cm}^{-3}$, so that the number densities associated with warm dark matter are still very low indeed. We wish to consider whether the value of $n_{e}$ can yield to experimental manipulation. In particular, we would like to increase the dark-matter number density in a particular region of momentum space. At first glance this would seem impossible [126], because Liouville's theorem demands that the density of the phase-space fluid for a system governed by a Hamiltonian is a constant of motion. Yet the Maxwell-Boltzmann velocity distribution we assume, cum gravitational potential, is a solution of Liouville's equation, so that a modest increase of number density 
can be realized by reducing the gravitational potential. Significant gains, however, are also possible, if inelastic processes operate, as in this case Liouville's theorem no longer limits the density. Such considerations are crucial to the construction of superthermal, ultra-coldneutron sources [126]; however, adapting this technology to our current context does not appear to be practical.

\section{SUMMARY}

A Faraday effect also exists for light transiting a dark medium of electrically neutral particles with non-zero magnetic moments in an external magnetic field [19]. We have used this notion to describe a Faraday rotation experiment which can lead to the direct detection of dark matter were it to possess a magnetic moment $\mu$. Alternatively, a null result can be used to limit the magnetic moment of a dark matter particle. The set-up involves an evacuated optical cavity to which an external magnetic field has been applied and through which the light makes multiple passes in the direction of the magnetic field. We assume the dark matter wind in the Earth rest frame sweeps through the apparatus. The size of the Faraday rotation angle is proportional to the magnetization of the dark matter, so that it is important to give the magnetic moments some net alignment. For particles with sufficiently low kinetic energy, the passage into the magnetic field region itself acts as a longitudinal Stern-Gerlach device; the highest energy state in the magnetic field can be barred from entering the apparatus, engendering a net polarization. We note that such a technique has been used to polarize ultra-cold neutrons with near 100\% efficiency [23]. Employing the usual assumptions [96] concerning the mass density and velocity distribution of galactic dark matter employed in the analysis of existing direct detection experiments, we have estimated, for fixed astronomical input, the polarization of the dark matter in the magnetic field region as a function of its mass and magnetic moment, as well as of the applied magnetic field. Given a local dark-matter mass density, our limits on $|\mu|$ then follow from the strength of the applied magnetic field $B_{0}$ and from the ability to measure $\phi_{0} / l$, the Faraday rotation angle accrued per unit length.

We have studied a range of candidate masses compatible with dark matter as a warm thermal relic, namely, from $1 \mathrm{keV}$ to $100 \mathrm{MeV}$. This window in candidate masses is not accessible via other direct detection techniques and thus is unique to our study. We find the strongest limits on $|\mu|$ emerge at the lightest mass scales we consider. In setting our limits we have employed the specifics of existing, related experiments as far as possible [23, 36], though we note that the possibility of more precise polarimetry has already been demonstrated [123, 124]. The sensitivity of the limits we obtain are such that they constrain the possibility that dark matter is a composite with a magnetic moment, akin to a stable neutron without its strong interactions. Indeed this analogy has proven useful in adapting technology used to manipulate neutrons to our current case. The technical limits of the polarimetry measurements have not yet been established [124, 125], and mounting the experiment in matter with concomitant gains in the applied magnetic field may prove feasible, so that we can ultimately expect better limits on $|\mu|$ than those found in this paper. 


\section{ACKNOWLEDGMENTS}

I am grateful to Geoff Greene for helpful discussions and particularly for suggesting that dark matter candidates with a magnetic moment could be polarized with a longitudinal Stern-Gerlach device. I thank Dima Budker for helpful comments and correspondence and for bringing Ref. [33] to my attention. I also thank Jeff Nico and Tom Gentile for many tolerant discussions and much encouragement, as well as Bob Golub and Mike Romalis for helpful remarks. I would like to thank the SLAC theory group, the Institute for Nuclear Theory, the Kavli Institute for Theoretical Physics, as well as the Center for Particle Astrophysics and Theoretical Physics at Fermilab, for gracious hospitality and to acknowledge partial support from the U.S. Department of Energy under contract DE-FG02-96ER40989, and from the National Science Foundation under Grant No. NSF PHY05-51164.

[1] S. M. Faber and J. S. Gallagher, Ann. Rev. Astron. Astrophys. 17, 135 (1979).

[2] A. Bosma, Astron. J. 86, 1825; V. C. Rubin, N. Thonnard, and W. K. . Ford, Astrophys. J. 238, 471 (1980); V. C. Rubin, D. Burstein, W. K. . Ford, and N. Thonnard, Astrophys. J. 289, 81 (1985); T. S. van Albada and R. Sancisi, Phil. Trans. R. Soc. Lond. A320, 447 (1986). For a review, see Y. Sofue and V. Rubin, Ann. Rev. Astron. Astrophys. 39, 137 (2001).

[3] D. J. Eisenstein and W. Hu, Astrophys. J. 496, 605 (1998); D. J. Eisenstein et al. [SDSS Collaboration], Astrophys. J. 633, 560 (2005). For a review, see R. C. Nichol, Gen. Rel. Grav. 40, 249 (2008).

[4] W. Hu and N. Sugiyama, Astrophys. J. 444, 489 (1995); Phys. Rev. D 51, 2599 (1995); G. Jungman, M. Kamionkowski, A. Kosowsky, and D. N. Spergel, Phys. Rev. D 54, 1332 (1996); M. Zaldarriaga, D. N. Spergel, and U. Seljak, Astrophys. J. 488, 1 (1997). For a review, see W. Hu and S. Dodelson, Ann. Rev. Astron. Astrophys. 40, 171 (2002).

[5] D. N. Spergel et al. [WMAP Collaboration], Astrophys. J. Suppl. 148, 175 (2003); M. Tegmark et al. [SDSS Collaboration], Phys. Rev. D 69, 103501 (2004).

[6] D. Clowe, M. Bradac, A. H. Gonzalez, M. Markevitch, S. W. Randall, C. Jones, and D. Zaritsky, Astrophys. J. 648, L109 (2006).

[7] P. Zhang, M. Liguori, R. Bean, and S. Dodelson, Phys. Rev. Lett. 99, 141302 (2007).

[8] K. A. Olive, arXiv:astro-ph/0503065, as well as arXiv:astro-ph/0301505, and references therein.

[9] G. Bertone, D. Hooper, and J. Silk, Phys. Rept. 405, 279 (2005).

[10] S. D. M. White, C. S. Frenk, and M. Davis, Astrophys. J. 274, L1 (1983).

[11] J. R. Ellis, J. S. Hagelin, D. V. Nanopoulos, K. A. Olive, and M. Srednicki, Nucl. Phys. B 238, 453 (1984). See also H. Goldberg, Phys. Rev. Lett. 50, 1419 (1983).

[12] B. Holdom, Phys. Lett. B 178, 65 (1986); S. Dimopoulos, D. Eichler, R. Esmailzadeh, and G. D. Starkman, Phys. Rev. D 41, 2388 (1990) and references therein; A. De Rujula, S. L. Glashow, and U. Sarid, Nucl. Phys. B 333, 173 (1990).

[13] P. F. Smith and J. R. J. Bennett, Nucl. Phys. B 149, 525 (1979); P. F. Smith, J. R. J. Bennett, G. J. Homer, J. D. Lewin, H. E. Walford, and W. A. Smith, Nucl. Phys. B 206, 333 (1982); P. F. Smith, Contemp. Phys. 29, 159 (1988) and references therein; T. K. Hemmick et al., Phys. Rev. D 41, 2074 (1990); P. Verkerk, G. Grynberg, B. Pichard, M. Spiro, S. Zylberajch, 
M. E. Goldberg, and P. Fayet, Phys. Rev. Lett. 68, 1116 (1992); T. Yamagata, Y. Takamori, and H. Utsunomiya, Phys. Rev. D 47, 1231 (1993); P. Mueller, L. B. Wang, R. J. Holt, Z. T. Lu, T. P. O'Connor, and J. P. Schiffer, Phys. Rev. Lett. 92, 022501 (2004).

[14] A. Gould, B. T. Draine, R. W. Romani, and S. Nussinov, Phys. Lett. B 238, 337 (1990).

[15] J. Rich, R. Rocchia, and M. Spiro, Phys. Lett. B 194, 173 (1987); G. D. Starkman, A. Gould, R. Esmailzadeh, and S. Dimopoulos, Phys. Rev. D 41, 3594 (1990).

[16] The empirical and astrophysical constraints we cite apply to candidate particles of varying mass, but in any event only to candidates with masses in excess of $20 \mathrm{GeV}$. The nonobservation of long-range (Coulombic), dark matter self-interactions [17] can also be interpreted as evidence in favor of the electric neutrality of dark matter.

[17] D. N. Spergel and P. J. Steinhardt, Phys. Rev. Lett. 84, 3760 (2000).

[18] Note, e.g., P. Sikivie, Lect. Notes Phys. 741, 19 (2008), for a review.

[19] S. Gardner, Phys. Rev. Lett. 100, 041303 (2008).

[20] D. Polder, Phil. Mag. 40, 99 (1949).

[21] C. L. Hogan, Rev. Mod. Phys. 25, 253 (1953).

[22] S. J. Orfanidis, http://www.ece.rutgers.edu/orfanidi/ewa .

[23] B. Tipton et al., AIP Conf. Proc. 539, 286 (2000).

[24] A. Drukier and L. Stodolsky, Phys. Rev. D 30, 2295 (1984).

[25] P. F. Smith and J. D. Lewin, Phys. Rept. 187, 203 (1990).

[26] R. J. Gaitskell, Ann. Rev. Nucl. Part. Sci. 54, 315 (2004).

[27] R. Bernabei et al., Phys. Lett. B 389, 757 (1996).

[28] M. Pospelov and T. ter Veldhuis, Phys. Lett. B 480, 181 (2000).

[29] E. M. Drobyshevski, arXiv:0706.3095.

[30] R. Bernabei et al., Eur. Phys. J. C 53, 205 (2008).

[31] R. Bernabei et al. [DAMA Collaboration], arXiv:0804.2741 [astro-ph].

[32] F. Petriello and K. M. Zurek, arXiv:0806.3989 [hep-ph].

[33] I. M. Savukov, S.-K. Lee, and M. V. Romalis, Nature 442, 1021 (2006).

[34] D. Budker and M. V. Romalis, "Optical Magnetometry," Nature Physics 3, 227 (2007).

[35] R. Cameron et al., Phys. Rev. D 47, 3707 (1993).

[36] E. Zavattini et al. [PVLAS Collaboration], Phys. Rev. Lett. 96, 110406 (2006) [Erratum-ibid. 99, 129901 (2007)]; Phys. Rev. D 77, 032006 (2008).

[37] L. Roszkowski, Pramana 62, 389 (2004).

[38] The Dark Matter Scientific Assessment Group (DMSAG) A Joint Sub-panel of HEPAP and AAAC Report on the Direct Detection and Study of Dark Matter, July 5, 2007, http://www.science.doe.gov/hep/dmsagreportjuly18,2007.pdf, note Fig. 20.

[39] P. Blasi, S. Burles, and A. V. Olinto, Astrophys. J. 514, L79 (1999).

[40] S. D. M. White and M. J. Rees, Mon. Not. Roy. Astron. Soc. 183, 341 (1978).

[41] C. S. Frenk, S. D. M. White, and M. Davis, Astrophys. J. 271, 417 (1983).

[42] J. R. Bond, J. Centrella, A. S. Szalay, and J. Wilson, in Formation and Evolution of Galaxies and Large Structures in the Universe, ed. J. Andouze and J. Tran Thanh Van, (DordrechtReidel 1984), p. 87.

[43] M. Davis, G. Efstathiou, C. S. Frenk, and S. D. M. White, Astrophys. J. 292, 371 (1985).

[44] G. Kauffmann, Mon. Not. Roy. Astron. Soc. 281, 475 (1996); J. X. Prochaska and A. M. Wolfe, Astrophys. J. 487, 73 (1997); M. Rauch et al., arXiv:0711.1354 [astro-ph] and references therein.

[45] S. Tremaine and J. E. Gunn, Phys. Rev. Lett. 42, 407 (1979). 
[46] P. Bode, J. P. Ostriker, and N. Turok, Astrophys. J. 556, 93 (2001); B. Moore, T. Quinn, F. Governato, J. Stadel, and G. Lake, Mon. Not. R. Astron. Soc. 310, 1147 (1999); V. AvilaReese, P. Colin, O. Valenzuela, E. D’Onghia, and C. Firmani, Astrophys. J. 559, 516 (2001); M. Miranda and A. V. Macciò, arXiv:0706.0896v1.

[47] M. Viel, J. Lesgourgues, M. G. Haehnelt, S. Matarrese, and A. Riotto, Phys. Rev. D 71, 063534 (2005).

[48] U. Seljak, A. Makarov, P. McDonald, and H. Trac, Phys. Rev. Lett. 97, 191303 (2006).

[49] M. Viel, J. Lesgourgues, M. G. Haehnelt, S. Matarrese, and A. Riotto, Phys. Rev. Lett. 97, 071301 (2006).

[50] M. Viel, G. D. Becker, J. S. Bolton, M. G. Haehnelt, M. Rauch, and W. L. W. Sargent, Phys. Rev. Lett. 100, 041304 (2008).

[51] K. Petraki and A. Kusenko, Phys. Rev. D 77, 065014 (2008).

[52] S. Dodelson and L. M. Widrow, Phys. Rev. Lett. 72, 17 (1994).

[53] P. Hut, Phys. Lett. B 69, 85 (1977); B. W. Lee and S. Weinberg, Phys. Rev. Lett. 39, 165 (1977); M. I. Vysotsky, A. D. Dolgov and Y. B. Zeldovich, JETP Lett. 26, 188 (1977) [Pisma Zh. Eksp. Teor. Fiz. 26, 200 (1977)].

[54] J. L. Feng and J. Kumar, arXiv:0803.4196 [hep-ph].

[55] J. L. Feng, H. Tu, and H. B. Yu, arXiv:0808.2318 [hep-ph].

[56] W. Krolikowski, arXiv:0803.2977 [hep-ph].

[57] P. Hut and K. A. Olive, Phys. Lett. B 87, 144 (1979); D. V. Nanopoulos, D. Sutherland, and A. Yildiz, Lett. Nuovo Cim. 28, 205 (1980).

[58] K. Sigurdson, M. Doran, A. Kurylov, R. R. Caldwell, and M. Kamionkowski, Phys. Rev. D 70, 083501 (2004) [Erratum-ibid. D 73, 089903 (2006)].

[59] J. Knodlseder et al., Astron. Astrophys. 441, 513 (2005); P. Jean et al., Astron. Astrophys. 445, 579 (2006) and references therein.

[60] C. Boehm, D. Hooper, J. Silk, M. Casse, and J. Paul, Phys. Rev. Lett. 92, 101301 (2004); C. Boehm, P. Fayet, and J. Silk, Phys. Rev. D 69, 101302 (2004); D. Hooper, F. Ferrer, C. Boehm, J. Silk, J. Paul, N. W. Evans, and M. Casse, Phys. Rev. Lett. 93, 161302 (2004). Note also C. Boehm, T. A. Ensslin, and J. Silk, J. Phys. G 30, 279 (2004); C. Boehm and P. Fayet, Nucl. Phys. B 683, 219 (2004).

[61] J. F. Beacom, N. F. Bell, and G. Bertone, Phys. Rev. Lett. 94, 171301 (2005).

[62] J. F. Beacom and H. Yuksel, Phys. Rev. Lett. 97, 071102 (2006).

[63] D. Hooper, M. Kaplinghat, L. E. Strigari, and K. M. Zurek, Phys. Rev. D 76, 103515 (2007).

[64] D. Hooper and K. M. Zurek, Phys. Rev. D 77, 087302 (2008).

[65] R. Bernabei et al. [DAMA Collaboration], arXiv:0802.4336 [astro-ph].

[66] S. Khalil and O. Seto, arXiv:0804.0336 [hep-ph].

[67] G. Bertone, A. Kusenko, S. Palomares-Ruiz, S. Pascoli, and D. Semikoz, Phys. Lett. B 636, 20 (2006).

[68] W. Wang, C. S. J. Pun, and K. S. Cheng, Astron. Astrophys. 446, 943 (2006).

[69] D. P. Finkbeiner and N. Weiner, Phys. Rev. D 76, 083519 (2007); I. Cholis, L. Goodenough, and N. Weiner, arXiv:0802.2922 [astro-ph].

[70] M. Pospelov and A. Ritz, Phys. Lett. B 651, 208 (2007).

[71] H. Grotch and R. W. Robinett, Z. Phys. C 39, 553 (1988).

[72] N. Tanimoto, I. Nakano, and M. Sakuda, Phys. Lett. B 478, 1 (2000).

[73] R. Schwienhorst et al. [DONUT Collaboration], Phys. Lett. B 513, 23 (2001).

[74] N. Borodatchenkova, D. Choudhury, and M. Drees, Phys. Rev. Lett. 96, 141802 (2006). 
[75] W. J. Marciano, Phys. Rev. D 60, 093006 (1999) and references therein.

[76] S. Profumo and K. Sigurdson, Phys. Rev. D 75, 023521 (2007).

[77] J. Erler and P. Langacker, review in W. M. Yao et al. [Particle Data Group], J. Phys. G 33, 1 (2006).

[78] M. E. Peskin and D. V. Schroeder, An Introduction to Quantum Field Theory (AddisonWesley Publishing Company, Reading, MA, 1995).

[79] Our detailed expressions for $\Delta \hat{r}^{\text {new }}$ in Eqs. 445) disagree with those in Refs. [58, 76], and our numerical limits are more severe, in part because we employ the better known quantity $\Delta \hat{r}$, rather than $\Delta r$ [75, 77].

[80] G. G. Raffelt, Phys. Rept. 198, 1 (1990).

[81] A. Heger, A. Friedland, M. Giannotti, and V. Cirigliano, arXiv:0809.4703 [astro-ph] and references therein.

[82] A. D. Dolgov, Phys. Rept. 370, 333 (2002) and references therein.

[83] J. D. Jackson, Classical Electrodynamics, $3^{\text {rd }}$ ed. (John Wiley \& Sons, New York, 1999).

[84] B. F. Burke and F. Graham-Smith, An Introduction to Radio Astronomy, Second Edition (Cambridge University Press, Cambridge, 2002).

[85] L. I. Schiff, Quantum Mechanics, $3^{\text {rd }}$ ed. (McGraw-Hill Companies, New York, 1968).

[86] V. Bargmann, L. Michel, and V. L. Telegdi, Phys. Rev. Lett. 2, 435 (1959).

[87] H. Rauch and S. A. Werner, Neutron Interferometry: Lessons in Experimental Quantum Mechanics (Oxford University Press, Oxford, 2000), p. 74 and references therein.

[88] X. P. Hu and Y. Liao, Eur. Phys. J. C 53, 635 (2008).

[89] A. Abragam, The Principles of Nuclear Magnetism (Clarendon Press, Oxford, 1978), p. 2.

[90] I. I. Sobel'man, Introduction to the Theory of Atomic Spectra (Pergamon, Oxford, 1972), p. 331.

[91] E. Fermi and L. Marshall, Phys. Rev. 72, 1139 (1947).

[92] Note, e.g., http://www.magnet.fsu.edu/mediacenter/factsheets/records.html.

[93] E. M. Purcell and N. F. Ramsey, Phys. Rev. 78, 807 (1950).

[94] W. W. Havens, I. I. Rabi, and L. J. Rainwater, Phys. Rev. 72, 634 (1947).

[95] J. Q. Xia, H. Li, G. B. Zhao, and X. Zhang, arXiv:0708.1111 [astro-ph].

[96] S. R. Golwala, http://cosmology.berkeley.edu/preprints/cdms/golwalathesis/.

[97] J. D. Lewin and P. F. Smith, Astropart. Phys. 6, 87 (1996).

[98] A. M. Green, Phys. Rev. D 66, 083003 (2002).

[99] A. M. Green, Phys. Rev. D 68, 023004 (2003) [Erratum-ibid. D 69, 109902 (2004)].

[100] N. Fornengo and S. Scopel, Phys. Lett. B 576, 189 (2003).

[101] A. K. Drukier, K. Freese, and D. N. Spergel, Phys. Rev. D 33, 3495 (1986).

[102] F. J. Kerr and D. Lynden-Bell, Mon. Not. Roy. Astron. Soc. 221, 1023 (1986).

[103] C. Amsler et al., Phys. Lett. B667, 1 (2008).

[104] W. Dehnen and J. Binney, Mon. Not. Roy. Astron. Soc. 298, 387 (1998).

[105] J. Binney and M. Merrifield, Galactic Astronomy (Princeton University Press, Princeton, NJ, 1998) p. 30.

[106] C. J. Copi and L. M. Krauss, Phys. Rev. D 67, 103507 (2003).

[107] J. Binney and S. Tremaine, Galactic Dynamics (Princeton University Press, Princeton, NJ, 1987) p. 226.

[108] G. Jungman, M. Kamionkowski, and K. Griest, Phys. Rept. 267, 195 (1996).

[109] M. Kamionkowski and S. M. Koushiappas, Phys. Rev. D 77, 103509 (2008).

[110] J. Ellis, K. A. Olive, and C. Savage, Phys. Rev. D 77, 065026 (2008). 
[111] E. I. Gates, G. Gyuk, and M. S. Turner, Astrophys. J. 449, L123 (1995).

[112] M. Kamionkowski and A. Kinkhabwala, Phys. Rev. D 57, 3256 (1998).

[113] I. B. Khriplovich and E. V. Pitjeva, Int. J. Mod. Phys. D 15, 615 (2006).

[114] I. B. Khriplovich, Int. J. Mod. Phys. D 16, 1475 (2007).

[115] L. Iorio, JCAP 0605, 002 (2006).

[116] M. Sereno and Ph. Jetzer, Mon. Not. Roy. Astron. Soc. 371, 626 (2006).

[117] J. M. Frère, F. S. Ling, and G. Vertongen, Phys. Rev. D 77, 083005 (2008).

[118] X. Xu and E. R. Siegel, arXiv:0806.3767 [astro-ph].

[119] M. Makela, H. O. Back, D. Melconian, and B. Plaster, AIP Conf. Proc. 842, 808 (2006).

[120] Note, e.g., "standard solenoid magnets" at http://www.oxinst.com/.

[121] http://www.magnet.fsu.edu/magnettechnology/research/magnetprojects/index.html.

[122] D. Budker, private communication.

[123] D. Budker, D. F. Kimball, S. M. Rochester, V. V. Yashchuk, and M. Zolotorev, Phys. Rev. A 62, 043403 (2000).

[124] D. Budker, W. Gawlik, D. F. Kimball, S. M. Rochester, V. V. Yashchuk, and A. Weis, Rev. Mod. Phys. 74, 1153 (2002) and references therein.

[125] P. Grangier, R. E. Slusher, B. Yurke, and A. LaPorta, Phys. Rev. Lett. 59, 2153 (1987).

[126] R. Golub and J. M. Pendlebury, Rep. Prog. Phys. 42, 439 (1979). Note the discussion on p. 453 in regards to Liouville's theorem. 